\title{
Remote Sensing Image Registration Techniques: A Survey
}

\author{
Suma Dawn ${ }^{1}$, Vikas Saxena ${ }^{1}$, and Bhudev Sharma ${ }^{2}$ \\ ${ }^{1}$ Department of Computer Science Engineering and Information Technology \\ ${ }^{2}$ Department of Mathematics \\ Jaypee Institute of Information Technology, \\ A-10, Sector -62, Noida, U.P., India \\ \{suma.dawn, vikas.saxena, bhudev.sharma\}@jiit.ac.in
}

\begin{abstract}
Image Registration is the first step towards using remote sensed images for any purpose. Despite numerous techniques being developed for image registration, only a handful has proved to be useful for registration of remote sensing images due to their characteristic of being computationally heavy. Recent flux in technology has prompted a legion of approaches that may suit divergent remote sensing applications. This paper presents a comprehensive survey of such literatures including recently developed techniques.
\end{abstract}

Keywords: Remote Sensing Images, Image Registration, Feature selection, control Point matching.

\section{Introduction}

Image registration is the process of transforming the different set of data into one coordinate system; also, may be said as the process of overlaying two or more images of the same scene taken at different times, from different viewpoints or from different sensors. Its main aim is to geometrically align two images.

Despite numerous techniques being developed for image registration [1], only a few have proved to be useful for registration of remote sensing images due to their characteristic of being computationally heavy. Recent flux in technology has prompted a legion of approaches that may suit a particular remote sensing application. Registration is used in the first phase for analysis of images received from one or more sensors (multimodal) having variable spatial or temporal variations.

Major applications of remote sensing image registration are in the fields of medicine, cartography, climatology, archaeosurvey, hydrology and hydrogeology, pattern recognition, geographical information system etc [2], [4], [24], [38], [77].

The procedure followed for registering two remote sensed images of a given scene, as illustrated by Goshtasby [2], are: (a) Preprocessing, (b) Feature Selection, (c) Feature Correspondence, (d) Determination of a transformation function and (e) Resampling. Of these afore mentioned steps, feature selection, correspondence and the transfer function determination are ones in which numerous techniques, for manipulation, may be applied. It is these variations that form the basis of classification stated in this letter. 
Section 2 categorizes some of the recent trends and technologies for image registration. A comparative study is presented in section 3. Finally, section 4 concludes this letter.

\section{Classification of Image Registration Techniques}

Feature selection phase may be based on the orientation of particular structures (regions, lines, curves, corners, etc) present in the images or registration may be dependent on the geometric orientation of the pixels and their intensities. These catetories are - feature-based and intensity-based respectively.

Classical intensity-based methods for registering imagery are cross-correlation; normalized cross-correlation (NCC) and minimum distance criteria [3] wherein registration involves calculating a transformation between two images using the pixel or voxel values alone. In its purest form, the registration transformation is determined by iteratively optimizing some similarity measure calculated from all pixel or voxel values [86]. Ground Control points (GCP), as primarily used by feature-based registration routines, represent centers of unique neighbourhoods that contain considerable image information [2]. Most of these are culminated and used in by Mohr [37], Brown [4], and Flusser and Zitova [5].

Bases on the evolution of techniques and minor deviations being incorporated by these registration styles, we have categorized the literatures as per their core concepts used for feature selection or correspondence or transformation functions determination. The classes are as follows:

\section{Class 1 - Similarity-metrics based methods.}

Similarity-metrics like Mutual Information, Correlation Coefficient, and histogram among others, though traditionally used as a measure to determine the correctness of registered images has, altogether, evolved as a method for registration of images. Correlation based similarity measure is a basic statistical approach and is useful for images which are misaligned by small rigid or affine transformations [59]. Mutual Information has found acceptance both in the fields of geosciences applications and medical images, because of its generality and high accuracy [34],[76], Arya et al [32], Chen et al [39], and Boes and Meyer [43]. Li et al [63] presented an automated intensity-based image registration approaches namely the simultaneous perturbation stochastic approximation (SPSA) algorithm. Xie et al [40] analyzed the two interpolation algorithms, namely bilinear interpolation and partial volume interpolation. A new indicator - misregistration value - was proposed to assess registration accuracy based on estimated offset between the corresponding points in the principal and the candidate image by spectral diversity algorithm by Lu et al [64].

\section{Class 2 - Methods using a combination of Simulated Annealing and Genetic algorithms.}

Yagouni [6] had proposed a rigid registration technique for 2 monomodal image which adapted two optimization metaheuristics, the Simulated Annealing and Genetic Algorithms, in their sequential and parallel versions. A combination of Simulated 
Annealing based Marquardt-Levenberg (SA-ML) with mutual information together with a wavelet based multiresolution pyramid due to Simoncelli was introduced by Ghorbani et al [70]. Genetic Algorithm application for image registration was also explored by Seixas et al [73] who addressed the point matching problem by employing a method based on nearest-neighbor.

\section{$\underline{\text { Class } 3}$ - Pyramid or triangular and Coarse-to-Fine method based.}

Massout et al [24] inducted a method based on pyramid algorithms and morphological filtering to create synthesis images having both high spatial resolution and high spectral resolution. To algorithmically improve registration time, a pyramid scheme to provide coarse-to-fine grain registration process was employed by Bui et al [9].

Xuehua et al [13] used a combination of Moravec and Forstner operator for feature point matching. Coarse-to-fine matching process was also used by Chen et al [31] in which initially, the image pyramid of working and reference images were constructed followed by feature points matching. Yulin et al [52] purported a coarseto-fine matching by incorporating an edge feature consensus method to rectify the rotation and translation difference between the SAR images globally in the coarse level registration; and automatically rectifying global control point pairs form the edge maps. Another multi-source image registration using coarse-to-fine method was also proposed by Zhang et al [56]. Lu et al [33] proposed another coarse-to-fine registration framework using Quantum Particle Swarm Optimization (QPSO) as optimizer. Quick and robust geometric registration of parallax image using coarse-tofine strategy was proposed by Junbin et al [65].

Class 4 - Methods using points, edges, corners, road-junctions and road point extraction as basis.

Man-made structures are proficient for depiction of straight lines, junctions, T-points, etc. For registration of city images via remote sensing, straight lines are used as an important feature by Bentoutou et al [46], Lou et al. [58], Zou et al [28], Dell'Acqua et al [22], Xiong et al [29], Alkaabi et al [51], Wen et al [74] and Weijie et al [14]. Zhang [12] used Radon transform as a tool for straight line detection. Bilinear interpolation and road networks based GCPs, which are the characters of urban remote sensing, were used by Guo et al [45] and Li et al [7]. Normalized sum of absolute differences metric (NSAD) based method was used by Alkaabi et al [51].

Multicamera registration and mosaicking method using control points and projective transformation was shown by Holtkamp et al [27]. Automatic Registration of Remote-Sensing Images (ARRSI), an automatic registration system built to register multimodal and multitemporal satellite and aerial remotely sensed images was developed by Wong et al [68] which used phase-congruency model and a variation on the Random Sample and Consensus algorithm called Maximum Distance Sample Consensus (MDSAC) for registration and was said to be capable of handling remotely sensed images geometrically distorted by various transformations such as translation, rotation, and shear.

$\underline{\text { Class } 5}$ - Segmentation, Point-Scatter and Learning-based methods. 
Pohl [38] justified using the segmented features of the image to generate and recursively evaluate a number of selected hypotheses as a basis for image registration especially for multiresolution images. Modified watershed transformation algorithm was used for image segmentation by Zhao et al [16] for feature-based geometric registration. Goncalves et al [17] also proposed several measures for an objective evaluation of the geometric correction process. Similar measures were also proposed by Aldrighi et al [18] wherein they used mode-based feature matching scheme which was initially used for computer vision application but latter adapted to pre- and postevent matching. Another novel algorithm for registration of SAR images was offered by Kang [61]. Isolated point scatterers (IPS) was used by Serafino et al [62]. Baboulaz [8] took maximum advantage of the a priori knowledge of the acquisition filter for feature extraction.

Barbu et al [21] proposed a system to learn the registration algorithm using a larger pool of feature-based registration algorithms from which a small number was selected and combined using AdaBoost. The pool of weak registration algorithms from which the boosted algorithm will be trained contains phase-correlation based algorithms and feature-based registration algorithms. A neural computational approach was presented by Srikanchana et al [42] which used a mixture of principal axes registration (mPAR) method and a multilayer perceptron neural network (MLP) to recover the nonlinear deformation.

\section{Class 6 - Fourier transformation and wavelet based techniques.}

The Fourier-based method works with the images in the frequency domain and utilizes the translation and rotational properties of Fourier transform to calculate the transformation parameters as stated in Xu et al [19], Li [75]. Zhou et al [48] used the wavelet-based global image registration, which was also used by Hong et al in [44], and [69]. Phase correlation (PC) method usage was shown by Erives and Fitzgerald [57]. Zhou et al [35],[48] tested serial and parallel strategies of wavelet-base automatic image registration for ChinaGrid project. Xu et al [19] used the Multiple Signal Classifier (MUSIC) algorithm whereas Qumar et al [31] used Discrete Multiwavelet transform (DMWT) methods. Steerable Simoncelli filters was used by Li et al [71] and Plaza, Moigne and Netanyahu [54]. Kasetkasem [20], proposed the use of the particle filtering algorithm to search for the global optima. Mixel decomposition of remote sensing images using particle swarm intelligence searching method was proposed by Wang, Dong et al [26]. In another paper, Zavorin and Moigne [49] proposed a method to handle single- and multisensor satellite data with relatively small amounts of nonlinear radiometric variation.

\section{Class 7 - Miscellaneous Methods.}

A combination of area-based matching technique with feature-based matching technique is, many a times, used to register images. A hybrid procedure was developed by Jia et al [47], Li et al [50], and Jianying [66].

Cluster machines could be utilized for parallel implementation of mutual information based image registration, as presented by $\mathrm{Du}$ et al [55] which used conjugate-gradient methods implemented on Commercial off-the-shelf (COTS) cluster 
Table 1. Classification and comparison of techniques used for registration of remote sensing images based on the literature study

\begin{tabular}{|c|c|c|c|}
\hline 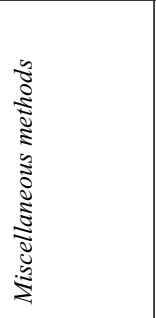 & 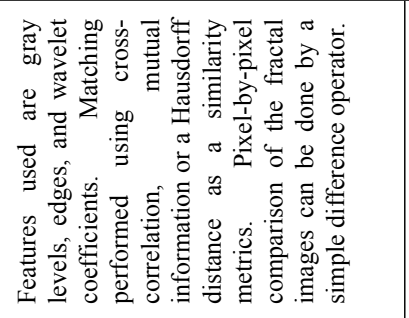 & 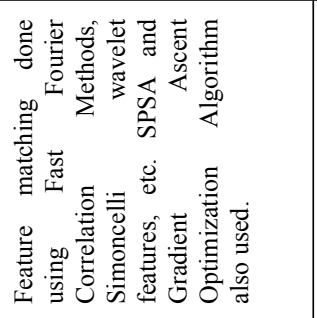 & 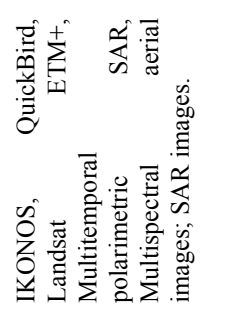 \\
\hline 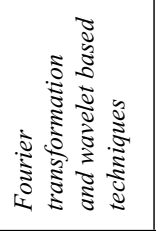 & 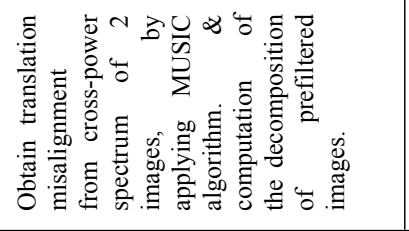 & 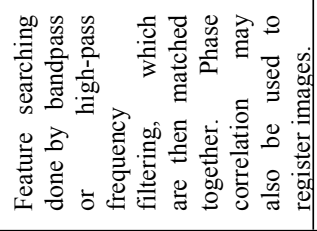 & 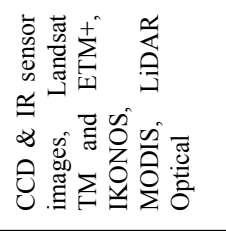 \\
\hline 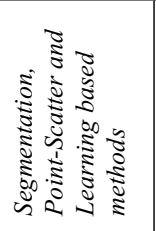 & 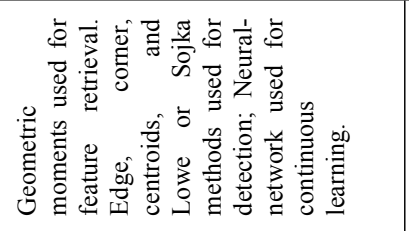 & 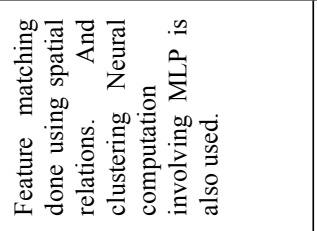 & 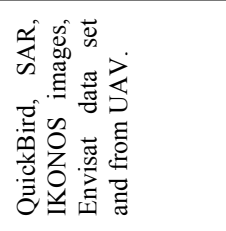 \\
\hline 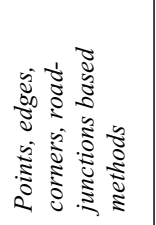 & 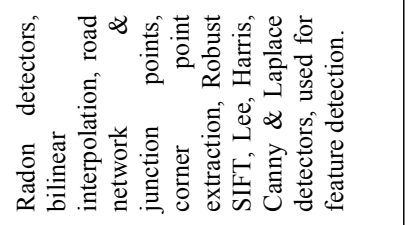 & 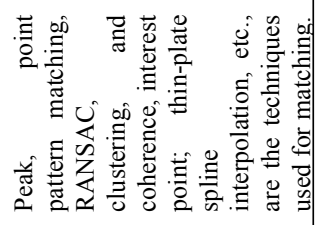 & 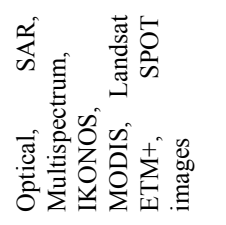 \\
\hline 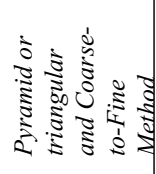 & 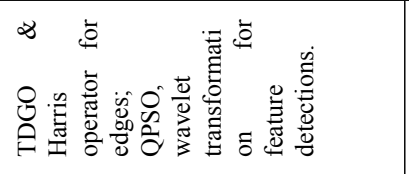 & 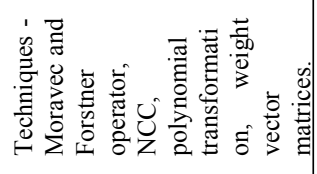 & 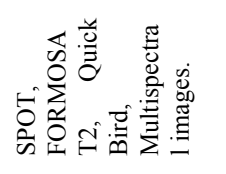 \\
\hline 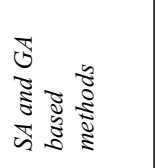 & 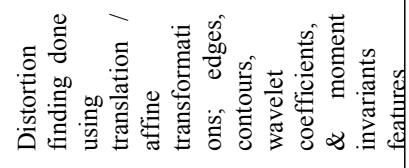 & 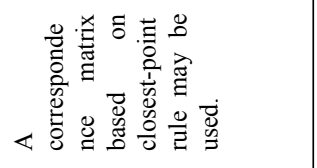 & 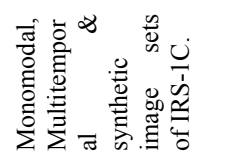 \\
\hline 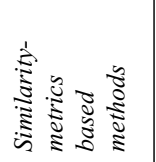 & 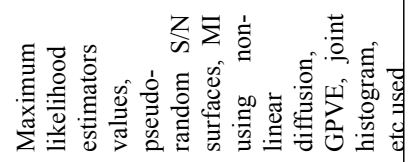 & 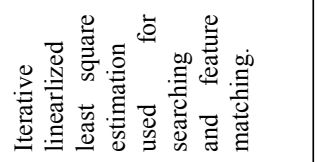 & 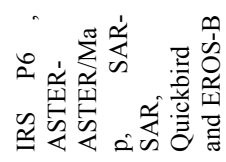 \\
\hline 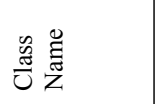 & 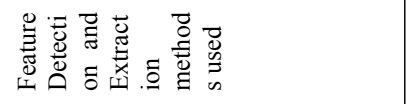 & 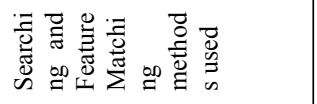 & 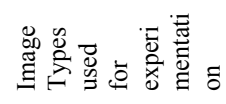 \\
\hline
\end{tabular}


of parallel Linux computer. Gupta et al [72] presented an efficient VLSI architecture for real-time implementation of image registration algorithms using an exhaustive search method.

Registration of images from GIS and remote sensing images may give erroneous results in the final registration result. Tian et al [53] suggested an automatic algorithm for the image-map registration problem using modified partial Hausdorff distance (MPHD) was proposed as a distance measure. Image registration may also be modeled by a displacement vector field that may be estimated by measuring rigid local shifts for each pixel in the image as shown by Inglada [67].

Tzeng et al [60] used Fractal method that could be applied to multitemporal polarime tric SAR images for change detections. Mitray et al [41] used point cloud data (PCD).

\section{Comparative Study}

The various classes described in this letter has been presented in the tabular format in Table 1, wherein not only are the main feature detection and extraction methods along with searching and feature matching methods have been illustrated but also the types of images mostly used for experimentation by these methods have also been discussed.

\section{Conclusion}

In this letter, we have collected together methods which have been classified and categories by considering the core theme or procedure used for registration. Recent techniques presenting a hybrid method seemed to be giving better results, as shown by the experimentation done by various authors, for the required application satisfaction. Hence, many a methods are not generally classified on the basis of the key classes of image registration methods, rather is done on the mathematical and statistical methodology used in the background for the registration. Some of the work may be further enhanced in the directions of providing a better framework for encompassing umpteen applications.

\section{References}

[1] Fonseca, L.M.G., Manjunath, B.S.: Registration Techniques for Multisensor Remotely Sensed Imagery. Photogrammetric Engineering \& Remote Sensing 62(9), 1049-1056 (1996)

[2] Goshtasby, A.: 2-D and 3-D Image Registration for Medical, Remote Sensing, and Industrial Applications. Wiley-Interscience Publication, Hoboken (2005)

[3] Webber, W.F.: Techniques for Image Registration. In: Conference on Machine Processing of Remotely Sensed Data (1973)

[4] Brown, L.G.: A Survey of Image Registration Techniques. ACM Computing Surveys 24, 326-376 (1992)

[5] Zitova, B., Flusser, J.: Image Registration Methods: A Survey. Image and Vision Computing 21, 977-1000 (2003)

[6] Yagouni, M.: Using Metaheuristics for Optimizing Satellite Image Registration. In: International Conference on Computers \& Industrial Engineering (2009) 
[7] Qiaoliang, L., Wang, G., Liu, J., Chen, S.: Robust Scale-Invariant Feature Matching for Remote Sensing Image Registration. IEEE Geoscience and Remote Sensing Letters 6(2), 287-291 (2009)

[8] Baboulaz, L., Dragotti, P.L.: Exact Feature Extraction Using Finite Rate of Innovation Principles with an Application to Image Super-Resolution. IEEE Transactions on Image Processing 18(2), 281-298 (2009)

[9] Bui, P., Brockman, J.: Performance Analysis of Accelerated Image Registration Using GPGPU. In: Proceedings of 2nd Workshop on GPGPU (2009)

[10] Wang, W., Liu, Y., Zheng, B., Lu, J.: A Method of Shape Based Multi-Sensor Image Registration. Joint Urban Remote Sensing Event 1-5 (2009)

[11] Yang, J., Zhao, Z.: A New Image Feature Point Detection Method Based on Log-Gabor Gradient Feature. Joint Urban Remote Sensing Event, 1-5 (2009)

[12] Jun, Z.: A Study on Automated Image Registration Based on Straight Line Features. Joint Urban Remote Sensing Event, 1-6 (2009)

[13] Lin, X., Yongzhi, Z., Jiazi, Y.Y.: Application of Triangulation-Based Image Registration Method in the Remote Sensing Image Fusion. In: International Conference on Environmental Science and Information Application Technology, vol. 1, pp. 501-504 (2009)

[14] Weijie, J., Jixian, Z., Jinghui, Y.: Automatic Registration of SAR and Optics Image Based on Multi-Feature on Suburban Areas. Joint Urban Remote Sensing Event, 1-7 (2009)

[15] Serief, C., Bentoutou, Y., Barkat, M.: Automatic Registration of Satellite Images. In: First International Conference on Advances in Satellite and Space Communications, pp. 85-89 (2009)

[16] Zhao, Y., Liu, S., Du, P., Li, M.: Feature-Based Geometric Registration of High Spatial Resolution Satellite Imagery. Joint Urban Remote Sensing Event, 1-5 (2009)

[17] Goncalves, H., Goncalves, J.A., Corte-Real, L.: Measures for an Objective Evaluation of the Geometric Correction Process Quality. IEEE Geoscience and Remote Sensing Letters 6(2), 292-296 (2009)

[18] Aldrighi, M., Dell'Acqua, F.: Mode-Based Method for Matching of Pre- and Postevent Remotely Sensed Images. IEEE Geoscience and Remote Sensing Letters 6(2), 317-321 (2009)

[19] Xu, M., Varshney, P.K.: A Subspace Method for Fourier-Based Image Registration. IEEE Geoscience and Remote Sensing Letters 6(3), 491-494 (2009)

[20] Kasetkasem, T., Homsup, N., Meetit, D.: An Image Registration Algorithm Using Particle Filters. In: 6th International Conference on Electrical Engineering/ Electronics, Computer, Telecommunications and Information Technology, vol. 02, pp. 1120-1123 (2009)

[21] Barbu, A., Ionasec, R.: Boosting Cross-Modality Image Registration. Joint Urban Remote Sensing Event, 1-7 (2009)

[22] Dell'Acqua, F., Sacchetti, A.: Steps towards a New Technique for Automated Registration of Pre- and Post-Event Images. Joint Urban Remote Sensing Event, 1-5 (2009)

[23] Liying, W., Weidong, S.: A Review of Range Image Registration Methods with Accuracy Evaluation. Joint Urban Remote Sensing Event, 1-8 (2009)

[24] Massout, S., Smara, Y., Ouarab, N.: Comparison of Fusion by Morphological Pyramid and By High Pass Filtering. Joint Urban Remote Sensing Event, 1-6 (2009)

[25] McCartney, M.I., Zein-Sabatto, S., Malkani, M.: Image Registration for Sequence of Visual Images Captured by UAV. In: IEEE Symposium on Computational Intelligence for Multimedia Signal and Vision Processing, pp. 91-97 (2009) 
[26] Dong, W., Xiangbin, W., Dongmei, L.: Particle Swarm Mixel Decomposition for Remote Sensing Images. In: IEEE International Conference on Automation and Logistics, pp. 212-216 (2009)

[27] Holtkamp, D.J., Goshtasby, A.A.: Precision Registration and Mosaicking of Multicamera Images. IEEE Transactions on Geoscience and Remote Sensing 47(10), 3446-3455 (2009)

[28] Zou, S., Zhang, J., Zhang, Y.: The Automatic Registration between High Resolution Satellite Images and a Vector Map Based on RFM. In: International Conference on Image Analysis and Signal Processing, pp. 397-401 (2009)

[29] Xiong, Z., Zhang, Y.: A Novel Interest-Point-Matching Algorithm for High-Resolution Satellite Images. IEEE Transactions on Geoscience and Remote Sensing (2009)

[30] Qumar, J., Pachori, R.B.: A Novel Technique for Merging of Multisensor and Defocussed Images using Multiwavelets. IEEE Region 10 TENCON, 1-6 (2005)

[31] Chen, C.F., Chen, M.H., Li, H.T.: Fully Automatic and Robust Approach for Remote Sensing Image Registration. In: Rueda, L., Mery, D., Kittler, J. (eds.) CIARP 2007. LNCS, vol. 4756, pp. 891-900. Springer, Heidelberg (2007)

[32] Arya, K.V., Gupta, P., Kalra, P.K., Mitra, P.: Image Registration Using Robust MEstimators. Pattern Recognition Letters 28, 1957-1968 (2007)

[33] Lu, Y., Liao, Z.W., Chen, W.F.: An Automatic Registration Framework using Quantum Particle Swarm Optimization for Remote Sensing Images. In: Proceedings of the 2007 International Conference on Wavelet Analysis and Pattern Recognition (2007)

[34] Wang, X., Zhang, J., Zhang, Y.: Registration of Remote Sensing Images Based on Gaussian Fitting. In: 3rd IEEE Conference on Industrial Electronics and Applications, pp. 378-381 (2008)

[35] Zhou, H., Tang, Y., Yang, X., Liu, H.: Research on Grid-Enabled Parallel Strategies of Automatic Wavelet based Registration of Remote-Sensing Images and Its Application ChinaGrid. In: Fourth International Conference on Image and Graphics, pp. 725-730 (2007)

[36] Bunting, P., Lucas, R., Labrosse, F.: An Area based Technique for Image-to-Image Registration of Multi-Modal Remote Sensing Data. In: IEEE International Geoscience and Remote Sensing Symposium, vol. 5(7-11), pp. 212-215 (2008)

[37] Rohr, K.: Localization Properties of Direct Corner Detectors. Journal of Mathematical Imaging and Vision (4), 139-150 (1994)

[38] Pohl, C., Van Genderen, J.L.: Multisensor Image Fusion in Remote Sensing: Concepts, Methods and Applications. International Journal of Remote Sensing 19(5), 823-854 (1998)

[39] Chen, H.M., Varshney, P.K., Arora, M.K.: Performance of Mutual Information Similarity Measure for Registration of Multitemporal Remote Sensing Images. IEEE Transactions on Geosciece and Remote Sensing 41(11) (2003)

[40] Xie, H., Pierce, L.E., Ulaby, F.T.: Mutual Information Based Registration of SAR Images. In: IEEE International Geoscience and Remote Sensing Symposium Proceedings, vol. 6(6), pp. 4028-4031 (2003)

[41] Mitray, N.J., Gelfandy, N., Pottmannz, H., Guibasy, L.: Registration of Point Cloud Data from a Geometric Optimization Perspective. In: Eurographics Symposium on Geometry Processing (2004)

[42] Srikanchana, R., Xuan, J., Freedman, M.T., Nguyen, C.C., Wang, Y.: Non-Rigid Image Registration by Neural Computation. Journal of VLSI Signal Processing 37, 237-246 (2004)

[43] Boes, J.L., Meyer, C.R.: Multi-variate Mutual Information for Registration. In: Taylor, C., Colchester, A. (eds.) MICCAI 1999. LNCS, vol. 1679, pp. 606-612. Springer, Heidelberg (1999) 
[44] Hong, G., Zhang, Y.: The Image Registration Technique for High Resolution Remote Sensing Image in Hilly Area. In: International Society of Photogrammetry and Remote Sensing Symposium (2005)

[45] Guo, X., Zhang, W., Ma, G.: Automatic Urban Remote Sensing Images Registration Based on Road Networks. IEEE Urban Remote Sensing Joint Event (2009)

[46] Bentoutou, Y., Taleb, N., Kpalma, K., Ronsin, J.: An Automatic Image Registration for Applications in Remote Sensing. IEEE Transactions on Geoscience and Remote Sensing 43(9), 2127-2137 (2005)

[47] Jia, X.: Automatic Ground Control Points Refinement for Remote Sensing Imagery Registration. In: Proceedings from International Conference on Intelligent Sensors, Sensor Networks and Information Processing Conference, pp. 145-149 (2005)

[48] Zhou, H., Yang, X., Liu, H., Tang, Y.: First Evaluation of Parallel Methods of Automatic Global Image Registration Based on Wavelets. In: International Conference on Parallel Processing, pp. 129-136 (2005)

[49] Zavorin, I., Le Moigne, J.: Use of Multiresolution Wavelet Feature Pyramids for Automatic Registration of Multisensor Imagery. IEEE Transactions on Image Processing 14(6), 770-782 (2005)

[50] Li, Y., Davis, C.H.: A Combined Global and Local Approach for Automated Registration of High Resolution Satellite Images Using Optimum Extrema Points. In: IEEE International Geoscience and Remote Sensing Symposium, vol. 2(2), pp. 1032-1035 (2008)

[51] Alkaabi, S., Deravi, F.: A New Approach to Corner Extraction and Matching for Automated Image Registration. In: Proceedings of IEEE International Geoscience and Remote Sensing Symposium, vol. 5, pp. 3517-3521 (2005)

[52] Yulin, Zhimin, Z., Wenge, C., Tian, J.: A New Registration Method for Multi-Spectral SAR Images. In: Proceedings of IEEE International Geoscience and Remote Sensing Symposium, vol. 3, pp. 1704-1708 (2005)

[53] Tian, L., Kamata, S., Ueshige, Y., Kuroki, Y.: An Automatic Image-Map Registration Algorithm Using Modified Partial Hausdorff Distance. In: Proceedings of IEEE International Geoscience and Remote Sensing Symposium, vol. 5, pp. 3534-3537 (2005)

[54] Plaza, A., Le Moigne, J., Netanyahu, N.S.: Automated Image Registration Using Morphological Region of Interest Feature Extraction. In: International Workshop on the Analysis of Multi-Temporal Remote Sensing Images, pp. 99-103 (2005)

[55] Du, Y., Zhou, H., Wang, P., Yang, X., Liu, H.: A Parallel Mutual Information Based Image Registration Algorithm for Applications in Remote Sensing. In: Guo, M., Yang, L.T., Di Martino, B., Zima, H.P., Dongarra, J., Tang, F. (eds.) ISPA 2006. LNCS, vol. 4330, pp. 464-473. Springer, Heidelberg (2006)

[56] Zhang, D., Yu, L., Cai, Z.: A Matching-Based Automatic Registration for Remotely Sensed Imagery. In: IEEE International Conference on Geoscience and Remote Sensing Symposium, pp. 956-959 (2006)

[57] Erives, H., Fitzgerald, G.J.: Automatic Subpixel Registration for a Tunable Hyperspectral Imaging System. IEEE Geoscience and Remote Sensing Letters 3(3), 397-400 (2006)

[58] Lou, X., Huang, W., Fu, B., Teng, J.: A Feature-Based Approach for Automatic Registration of NOAA AVHRR Images. In: IEEE International Conference on Geoscience and Remote Sensing Symposium, pp. 995-998 (2006)

[59] Martinez, A., Garcia-Consuegra, J., Abad, F.: A Correlation-Symbolic Approach to Automatic Remotely Sensed Image Rectification. In: IEEE International Geoscience and Remote Sensing Symposium, vol. 1, pp. 336-338 (1999) 
[60] Tzeng, Y.C., Chiu, S.H., Chen, K.S.: Automatic Change Detections from SAR Images Using Fractal Dimension. In: IEEE International Conference on Geoscience and Remote Sensing Symposium, pp. 759-762 (2006)

[61] Kang, X., Han, C., Yang, Y.: Automatic SAR Image Registration by Using Element Triangle Invariants. In: IEEE 9th International Conference on Information Fusion, pp. 1-7 (2006)

[62] Serafino, F.: SAR Image Coregistration Based on Isolated Point Scatterers. IEEE Geoscience and Remote Sensing Letters 3(3), 354-358 (2006)

[63] Li, Q., Sato, I., Murakami, Y.: Simultaneous Perturbation Stochastic Approximation Algorithm for Automated Image Registration Optimization. In: IEEE International Conference on Geoscience and Remote Sensing Symposium, pp. 184-187 (2006)

[64] Lu, B., Yanping, W., Lideng, W., Wen, H., Hailiang, P.: InSAR Co-registration Accuracy Assessment Based on Misregistration Value. In: IEEE International Conference on Geoscience and Remote Sensing Symposium, pp. 964-967 (2006)

[65] Junbin, G., Xiaosong, G., Wei, W., Pengcheng, P.: Geometric Global Registration of Parallax Images Based on Wavelet Transform. In: 8th International Conference on Electronic Measurement and Instruments (2007)

[66] Jianying, J., Qiming, Z.: A Novel Method for Multispectral Aerial Image Registration. In: IEEE International Geoscience and Remote Sensing Symposium, pp. 385-388 (2007)

[67] Inglada, J., Muron, V., Pichard, D., Feuvrier, Y.: Analysis of Artifacts in Subpixel Remote Sensing Image Registration. IEEE Transactions On Geoscience And Remote Sensing 45(1) (2007)

[68] Wong, A., Clausi, D.A.: ARRSI: Automatic Registration of Remote-Sensing Images. IEEE Transactions on Geoscience and Remote Sensing, Part 2 45(5), 1483-1493 (2007)

[69] Hong, G., Zhang, Y.: Combination of Feature-Based and Area-Based Image Registration Technique for High Resolution Remote Sensing Image. In: IEEE International Geoscience and Remote Sensing Symposium, pp. 377-380 (2007)

[70] Ghorbani, H., Beheshti, A.A.: Multiresolution Registration of Multitemporal Remote Sensing Image Optimization of Mutual Information Using a Simulated Annealing Based Marquardt-Levenberg Technique. In: International Conference on Intelligent and Advanced Systems, pp. 685-690 (2007)

[71] Li, Q., Sato, I., Murakami, Y.: Steerable Filter Based Multiscale Registration Method for JERS-1 SAR and ASTER Images. In: IEEE International Geoscience and Remote Sensing Symposium, pp. 381-384 (2007)

[72] Gupta, N., Gupta, N.: A VLSI Architecture for Image Registration in Real Time. IEEE Transactions On Very Large Scale Integration (VLSI) Systems 15(9), 981-989 (2007)

[73] Seixas, F.L., Ochi, L.S., Conci, A., Saade, D.C.M.: Image Registration Using Genetic Algorithms. In: Genetic and Evolutionary Computation Conference, GECCO’08, July 12-16 (2008)

[74] Wen, G.J., Lv, J.J., Yu, W.X.: A High-Performance Feature-Matching Method for Image Registration by Combining Spatial and Similarity Information. IEEE Transactions on Geoscience and Remote Sensing, Part 2 46(4), 1266-1277 (2008)

[75] Li, Q., Sato, I., Sakuma, F.: A Novel Strategy for Precise Geometric Registration of GIS and Satellite Images. In: IEEE International Geoscience and Remote Sensing Symposium, vol. 2(2), pp. 1092-1095 (2008)

[76] Bunting, P., Lucas, R., Labrosse, F.: An Area based Technique for Image-to-Image Registration of Multi-Modal Remote Sensing Data. In: IEEE International Geoscience and Remote Sensing Symposium, vol. 5(5), pp. 212-215 (2008)

[77] Ranganath, R.N., Jayaraman, V., Roy, P.S.: Remote Sensing Applications: An Overview. Current Science 93(12) (2007) 